\title{
Sarcopenia, Frailty and Dismobility
}

\author{
Edit Vereckei ${ }^{1}$, Andrea Ildikó Gasparik ${ }^{2}$ and László Hodinka*1 \\ ${ }^{1}$ National Institute of Rheumatology and Physiotherapy, Budapest, Hungary \\ ${ }^{2}$ University of Medicine and Pharmacy Tirgu Mures, Tirgu Mures, Romania
}

Received: 阱 July 17, 2018; Published: 阱July 25, 2018

*Corresponding author: László Hodinka, National Institute of Rheumatology and Physiotherapy, Budapest, Hungary H-1023 Budapest Frankel Leó u. 25-29.

\section{Abstract}

Sarcopenia is the progressive involution of musculature, with a decrease of power and with limitation of physical and cognitive functions, including isolation, loss of independence and impairment of the quality of life. It is a gerontology syndrome, a component of age-related frailty. Because of the dismobility there is a hazard of falls and fractures with a significant mortality risk. This article reviews the relation of sarcopenia to aging, definition and epidemiology and pathophysiology of sarcopenia with publications and recommendations for screening, diagnostic tools and therapeutic modalities.

Abbreviations: BMI: body mass index; EuroQoL-5D, EQ-5D: European Quality of Life 5 Dimension Questionnaire; EWGSOP: European Working Group on Sarcopenia in Older People; HRQoL: Health Related Quality of Life; SarQoL: Sarcopenia Quality of Life Questionnaire; SF-36: Rand Corporation Short Form Questionnaire 36 question version SMM... skeletal muscle mass; and skeletal muscle mass index, SMI: skeletal muscle mass index; SPPB: Short Physical Performance Battery.

\section{Introduction}

Sarcopenia is a progressive loss of muscle mass and power because of the gradual disintegration of the locomotor musculature becoming more prominent in older age. Sarcopenia results in the progressive limitation of muscular functions, first of all mobility, consequently the daily life activities and participation in the family and social life. The affected persons become isolated and need supportive care and are at risk of falls, low energy bone fractures and other traumas. These conditions lead to a decrease of cognitive abilities, anxiety and depression. With several possible age-related co-morbidities fundamentally determines the quality of life and means a significant burden for the affected individuals and for their families as the demographic trends show the growing elderly populations, sarcopenia is an emerging serious public health concern. This burden may be reduced by the early recognition of sarcopenia and its progression may be retarded with protective measures.

\section{Ageing and Sarcopenia}

The phenomenon of sarcopenia appeared first in the gerontology literature in 1987. After several symptomatic descriptions the first systematic attempt to describe the possible causes and mechanisms and defined this complex process as a loss of balance between synthesis and degradation of muscle proteins [1]. The question remains constant: till what sarcopenia can be an obligatory part of the physiological aging and from should it be considered a disease. The physiological reserve of the musculature is decreasing with the "successful aging", however in "accelerated aging" the simple degradation becomes a constituent of the physical frailty and with further progression leads to disability and to the loss of independence [2]. In the age-related frailty sarcopenia represents the poor muscle quality, resulting in weak muscle strength, slow gait and poor balance and these are added to the frailty complex, as accumulated deficits: weight loss, fatigue, inactive behavior, cognitive impairment and social isolation [3]. During the life course early life years are characterized with the growth of muscle mass and power to maximize the peak, adults should maintain them. In older life efforts should be made to minimize the loss among even very different individual conditions [4].

\section{Definition and epidemiology}

According to the recent European consensus of The European Working Group on Sarcopenia in Older People (EWGSOP) the diagnostic criteria of sarcopenia are: low muscle mass and at least one of the two further, low muscle strength and/o low physical performance. The cut-off points of these are determined in algorithms. In all the three stages of sarcopenia the low muscle mass should be present, in presarcopenia low muscle mass only, in sarcopenia low strength or weak performance added, in severe sarcopenia is characterized by all three criteria [5]. However, a meta-analysis showed, that average values of criteria elements may vary according to populations studies and methods used [6]. This is reflected in the multi-continental epidemiological study including 18363 people over 65 years in surveys conducted in 9 countries on five continents the prevalence of sarcopenia was found between 12,6 and 17,5 per cent, sarcopenic obesity (low muscle mass with high body fat) ranged from 1,3 to 11,0 per cent [7]. In the European 
BELFRAIL study using the EWGSOP criteria sarcopenia was found 12,5 per cent among very old (aged 80 years or more) [8].

\section{Pathophysiology of sarcopenia}

The EWGSOP consensus discriminates primary or agerelated sarcopenia with intrinsic causes and secondary caused by multiple dysfunctional mechanisms as basically endocrine, neuro-degenerative diseases, malnutrition or malabsorption, neoplastic cachexia and immobility, because of inactivity or even zero gravity [5]. Muscle development and regeneration are governed by a very wide variety of genetically determined factors, as myostatin, activin, insulin-like growth factor, ciliariry neurotropic factor, growth-differentiation factor, transforming growth factor, mechanical growth factor, etc. These may contribute to the normal or decreased synthesis of muscle proteins. Intact muscle cell mitochondrial functions are essential for the energy supply, and their age-related abnormalities are responsible for muscle involution $[9,10]$. Extrinsic factors playing a pathologic role in sarcopenia are e.g. atherosclerotic hypoxia, inflammatory cytokines, insulin resistance, increase of leptin, adipokines, playing a role also in increased fat infiltration and sarcopenic obesity [5].

\section{Screening}

"Red flags" and presenting features attracting attention on the suspected sarcopenia in the everyday practice or reported by the patient are general weakness, visible muscle loss, slow walking, decreased muscle strength, fatigue, difficulties in the activities or movements or daily living and falls [11]. Five clinical screening tools have been developed including the EWGSOP consensus algorithm. Further three have not been validated or use only insufficient indicators or are of little clinical utility. The SARC-F questionnaire is simple and has definite cut-offs [12]. The SARC-F consists of five self-answered questions on strength, walking, rise from a chair, climb stairs and falls and correlates fairly with several comparators [13].

\section{Diagnosis and clinical picture}

The diagnosis of sarcopenia relies upon the simple algorithm included into the EWGSOP consensus, with a hierarchy of age over 65 years, gait speed slower than $0,8 \mathrm{~m} / \mathrm{sec}$ and grip strength. lower than $30 \mathrm{~kg}$ at men and lower than $20 \mathrm{~kg}$ for women [5]. Further indicators are recommended by several authors for clinical practice and for research. These represent the muscle related clinical features of sarcopenia. For both practice and research anthropometric measurements (skeletal muscle mass, a composite index from weighed measures as weight, height, gender, age and ace, SMM, and skeletal muscle mass index, SMI, SMM/body mass index-BMI).and the Short Physical Performance Battery, SPPB (standing, tandem standing, gait speed and rise from a chair) $[14,15]$. Preferably for research bioelectric impedance, computed tomography, ultrasound, specially programmed dual energy X-ray absorption and magnetic resonance imaging are recommended, aiming the separate determination of muscle and fat composition $[5,6,16,17,18]$. Determination of several biomarkers are recommended: reactive oxygen species, protein carbonylation, advanced glycation products, AGEs, serum and urinary creatinine, N-terminal procollagen peptides, myostatin. dehydroepiandrosterone sulfate with testosterone and oestrogen [19]. Brain-derived neurotrophic factor is produced also in skeletal muscle and in myogenic progenitors and it may be. link between muscle and the central nervous system. Such connections may be present in the background of the sarcopenia-associated depression, anxiety and cognitive losses. [20, 21, 22]. Further significant comorbidity is diabetes and impaired glucose tolerance associated with insulin resistance. These are conditions promoting sarcopenic obesity. Cachexia caused by toxic tissue degradation products in neoplastic diseases and pro-inflammatory cytokines in chronic infections and therapy resistant autoinflammatory diseases as comorbid conditions associated with secondary sarcopenia enhance the risk of mortality in the affected mostly geriatric patients [23].

\section{Sarcopenia as a part of the dismobility syndrome}

Binkley, inspired by the striking similarities of age related bone and muscle involution and the association of other frailty markers, first fractures caused by falls suggested an overarching complex entity: the dysmobility syndrome [24]. Structured criteria for the classification of dysmobility syndrome requiring three or more present. as osteoporosis (T-score lower than $-2,5$ ), falls in the preceding year, low lean mass (less than $5,45 \mathrm{~kg} / \mathrm{m}^{2}$ men and $7,36 \mathrm{~kg} / \mathrm{m}^{2}$ women), low grip strength (less than $30 \mathrm{~kg}$ men and $20 \mathrm{~kg}$ women and high fat mass (over 30 per cent men and 40 for women) [25]. Presence of dismobility components derived from bone-muscle-adiposity measures show a significant hazard of mortality [26].

\section{Sarcopenia and Quality of Life}

All features of sarcopenia and related frailty and dismobility fundamentally impairs the quality of life of the affected. Several generic, osteoporosis and gerontology-specific quality of life questionnaires have been tested for the domains characterizing sarcopenia, However, these were not suitable for the proper reflection for sarcopenia specific patient reported outcomes [23]. This need led to the development of the Sarcopenia Quality of Life (SarQoL) questionnaire. This is a self-administered questionnaire of 22 questions with 55 preformed patient reported best fitting answers. The questioned domains are physical and mental health, locomotion, body composition, functionality, daily and leisure activities and fears [27]. The developers offered the English standard for translation according to the usual protocol and suggested its cross-cultural validation [28] One of the authors (AIG) had been authorized for the Romanian and Hungarian validation which was performed and published $[29,30]$.

\section{Treatment options}

Several treatment modalities have been proposed on theoretical and pathophysiological grounds (protein and amino acid supplementation, creatine, butyrate's, vitamins D and E, anabolic, androgens and its precursors, angiotensin convertase inhibitors, growth hormone, ghrelin agonists, adrenergic receptor, however there are no convincing evidence-based trials $[9,10,11,12]$. It is generally accepted that physical activity helps maintain muscular functions, aerobic and balance exercises a resistance training are of real therapeutic values [31]. 


\section{Conclusion}

Sarcopenia represents a significant disease and economic burden for the affected, for their caregivers and indirectly for the whole society. It is associated with the age-related frailty and the increased risk of falls, fractures, co-morbidities, impairs the quality of life and means a serious hazard of mortality. As it is progressive, emerges with the growing population worldwide. Early recognition of the sarcopenic state, identification of the possible causes, objective measurement of the multiple manifestations and timely and proper protective interventions are essential to prevent its dangerous complications.

\section{References}

1. Marcell T (2003) Review Article: Sarcopenia: causes, consequences, and preventions. J Gerontol a Biol Sci Med Sci 58: 911-916.

2. Calvani R, Marini F, Cesari M, Tosato M, Stefan DA, et al. (2015) Biomarkers for physical frailty and sarcopenia: state of the science and future developments. J Cachexia Sarcopenia and Muscle 6: 278-286.

3. Cesari M, Landi F, Vellas B, Roberto B, Emanuele M, (2014) Sarcopenia and physical frailty: two side of the same coin. Frontiers in Aging Neuroscience 6: 192

4. Dodds R, Sayer AA (2015) Sarcopenia and frailty: new challenges for clinical practice. Clin Med 15: 88-91.

5. Cruz Jentoft AJ, Baeyens JP, Bauer JM, Boirie Y, Cederholm T, et al. (2010) Sarcopenia: European consensus on definition and diagnosis. Age Ageing 39(4): 412-423.

6. Pagotto V, Sileira ES (2014) Methods, diagnostic criteria, Cutoff points and prevalence of sarcopenia among older people. The Scientific World Journal Article ID: 232312

7. Tyrovolas S, Koyanagi A, Olaya B, Jose Luis AM, Marta M, et al. (2016) Factors associated with skeletal muscle mass, sarcopenia, and sarcopenic obesity in older adults: a multi-continent study. J of Cachexia, Sarcopnia and Muscle 7: 312-321.

8. Legrand D, Vaes C, Matheï C, Swine C, Deqryse JM (2013) The prevalence of sarcopenia in very old individuals according to the Eufropean consensus definition: insights from the BELFRAIL study. Age and Ageing42: 727-734.

9. Morley JE (2016) Pharmacologic options for the treatment of sarcopenia. Calcif Tiss Int 8: 319-333.

10. Park SS, Kwon ES, Kwon KS (2017) Molecuar mechanisms and therapeutic interventions in sarcopenia. Osteoporosis and Sarcopenia 3: $117-122$.

11. Beaudart Ch, McCloskey E, Bruyère O, Matteo C, Yves R, et al. (2016) Sarcopenia in daily practice: assessment and management. BMC Geriatrics 16: 170

12. Yu SCY, Khow KSF, Jadczak AD et al. (2016) Clinical screening tools for sarcopenia and is management. Current Gerontology and Geriatrics Research :1-10.

13. Malmström TK, Miller DK, Simonsick EM, Ferrucci L, Morley JE (2016) SARC-F: a symptom score to predict persons with sarcopenia at risk for poor functional outcomes. J Cachexia Sarcopenia and Muscle 7: 28-36.

14. Lee RC, Wang ZM, Heo M, Ross M, Janssen I, et al. (2000) Totalbody skeletal muscle mass: development and cross-validation of anthropometric prediction models. Am J Clin Nutr 72(3): 796-803.
15. Kasper JD., Freedman VA, Niefeld R (2012) Construction of performancebased summary measures of physical capacity in the National Health and Aging Trends Study. NHATS Technical, Baltimore: Johns Hopkins University School of Public Health 212.

16. Rubbieri G, Mossello E, DiBari M (2014) Techniques for the diagnosis of sarcopenia. Clin Cases Mineral Bone Metabol 11: 181-184.

17. Gupta AK, Mishra S (2016) Sarcopenia and the syndrome of frailty. Egypt J Intern Med 28(4): 133-139.

18. West J, Leinhard OD, Romu T, Rory C, Steve G, et al. (2016) Feasibility of MR-based body composition analysis in large scale population studies. PLOS ONE 11(9).

19. Foguère B, Vellas B, van Kan GA, Cesari M (2015) Identification of biological markers for better characterization of older subjects wih physical frailty and sarcopenia. Translational Neuroscience 6(1): 103110.

20. Pasco JA, Williams LJ, Jacka FN, Stupka N, Holloway KL, et al. (2015) Sarcopenia and common mengtal disorders: a potential regulatory role of skeletal muscle on brain function. Curr Osteoporos Rep 13: 351-357.

21. Hamer M, Batty GD, Kivimaki M (2015) Sarcopenic obesity and risk of new onset depressive symptoms in older adults: English Longitudinal Study of Ageing. Int J 39(12): 1717-1720.

22. Tolea M, Galvin JE (2015) Sarcopenia and impairment in cognitive and physical performance. Clin Intervent Aging 10: 663-671.

23. Rizzoli R, Reginster JY, Arnal JF, Bautmans I, Beaudart C, et al. (2013) Quality of life in sarcopenia and frailty. Calcif Tissue Int 93: 101-120.

24. Binkley N, Krueger D, Buehring B (2013) What's in a name revisited: should osteoporosisand sarcopenia be considered components of “dysmobility syndrome?" Osteoporos Int 24: 2955-2959.

25. Hill KD, Farrier K, Russelll M, Burton E (2017) Dysmobility syndrome: current perspectives. Clinical Interventions in Aging 12: 145-152.

26. Lee WJ, Liu LK, Hwang AC, Peng LN, Lin MH, et al. (2017) Dysmobility syndrome and risk of mortality for community-dwelling middle-aged and older adults: the nexus of aging and body composition. Nature Scientific Reports 7: 8785.

27. Beaudart C, Biver E, Reginster JY, Rizzoli R, Rolland Y, et al. (2015) Development of a self- administrated quality of life questionnaire for sarcopenia in elderly subjects: the SarQoL. Age and Aging 44: 960-966.

28. Beaudart Ch, Reginster JY, Geerink A, Locquet M, Bruyere O (2017) Current review of the SarQoL : a health-related quality of life questionnaire specific to sarcopenia. Expert Review of Pharmacoeconomics Outcomes Research 17: 335-341.

29. Gasparik AI, Mihai G, Beaudart Ch, Olivier B, Maria PI, et al. (2017) Psychometric performance of the Romanian version of the SarQoL®, a health-related quality of life questionnaire for sarcopenia. Archives of osteoporosis 12(1): 103.

30. Hodinka L, Vereckei E, Gasparik AI (2018) Sarcopenia and quality of life: The validated Hungarian translation of the Sarcopenia Quality of Life (SarQoL) Questionnaire [Hungarian] Orv Hetil 159.

31. Hunt D, Chapa D, Hess B, Swanick K, Andrew H (2015) The importance of resistance training in the treatment of sarcopenia. J Nursing Educ Pract 5(3): 39-43. 
ISSN: 2574-1241

DOI: 10.26717/BJSTR.2018.07.001472

László Hodinka. Biomed J Sci \& Tech Res

CC (9) This work is licensed under Creative

Submission Link: https://biomedres.us/submit-manuscript.php

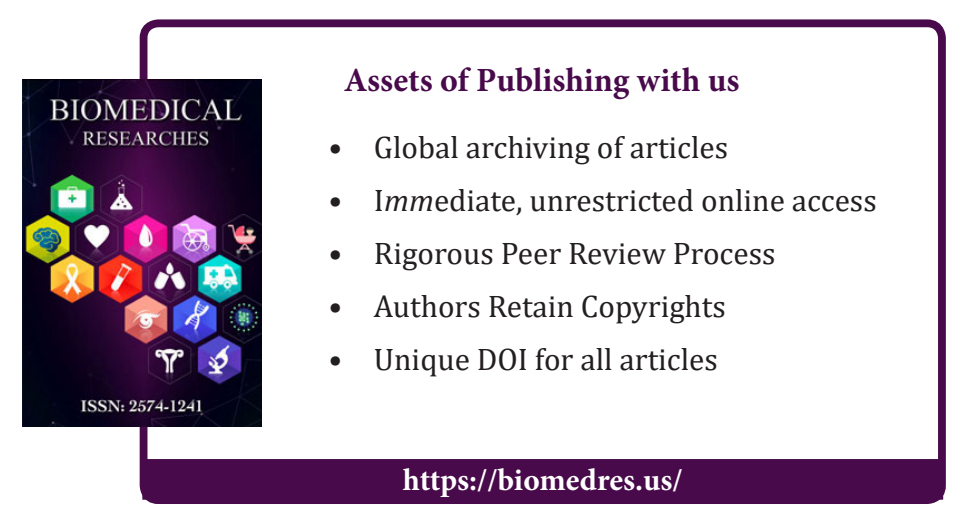

